INPLASY

PROTOCOL

To cite: Wang et al. Cefiderocol for the Treatment of MultidrugResistant Gram-Negative bacteria: A Systematic Review of Current Evidence. Inplasy protocol 2021100063. doi: 10.37766/inplasy2021.10.0063

Received: 18 October 2021

Published: 18 October 2021

Corresponding author: Wentao Ni

wentao.qingdao@163.com

Author Affiliation:

Peking University People's Hospital

Support: Research and Development Funds.

Review Stage at time of this submission: Preliminary searches.

Conflicts of interest:

None declared.

\section{Cefiderocol for the Treatment of Multidrug-Resistant Gram-Negative bacteria: A Systematic Review of Current Evidence}

\author{
Wang, C1; Yang, D2; Ni, W³.
}

Review question / Objective: In this review, we systematically searched and analyzed the current available literature to evaluate the role of cefiderocol in treating MDR Gramnegative bacteriainfections.

Information sources: We systematically searched the PUBMED, EMBASE and Cochrane Library databases. Besides, to reduce publication bias, we reviewed the conference proceedings of the International Symposium on Antimicrobial Agents and Resistance (ISARR), Infectious Diseases Society of America (IDSA) and European Congress of Clinical Microbiology and Infectious Diseases (ECCMID) from the years 2015-2021. We manually searched the reference lists of included studies and published reviews and systematic reviews for selecting relevant articles.

INPLASY registration number: This protocol was registered with the International Platform of Registered Systematic Review and Meta-Analysis Protocols (INPLASY) on 18 October 2021 and was last updated on 18 October 2021 (registration number INPLASY2021100063).

\section{INTRODUCTION}

Review question / Objective: In this review, we systematically searched and analyzed the current available literature to evaluate the role of cefiderocol in treating MDR Gram-negative bacteriainfections.
Rationale: The global spread of multi-drug resistant (MDR) bacteria has been a looming threat to the public health. In 2017, the WHO designated the ESKAPE pathogens (Enterococcus faecium, Staphylococcus aureus, Klebsiella pneumoniae, Acinetobacter baumannii, 
Pseudomonas aeruginosa, and Enterobacter species) as 'priority status', for which new antibiotics are urgently needed. Among the six ESKAPE pathogens, three Gram-negative bacteria usually with carbapenem-resistance are the most important superbugs in healthcare settings. They are frequently resistant to nearly all classes of antibiotics commonly used in clinical practice. Because of the limited therapeutic options, polymyxins, a class of cationic peptide drugs which was abandoned in last century due to high nephrotoxicity, has been reused to treat recalcitrant infections caused by carbapenem-resistant Gram-negative bacteria. However, the clinical outcome is unsatisfactory, and the mortality is persistently high among critically ill infected patients. Several new antibiotics that are discovering and developing in the pipeline or newly available in the market give us hope and confidence. All bacteria, especially the Gram-negative bacteria, need capture iron in the environment through siderophore-iron transport systems, as a cofactor in enzymes to catalyze redox reactions for various fundamental cellular processes. By taking advantage of this unique feature, a novel synthetic siderophore-conjugated antibiotic named cefiderocol has been developed, which can hijack the bacterial iron transport systems to facilitate the drug to entry into cells, achieving high periplasmic concentrations. Besides, cefiderocol has higher affinity for penicillin binding proteins 3 (PBP3), and the $\mathrm{C}-7$ side chain in cefiderocol improves transport across the bacterial outer membrane and resists the hydrolysis by several betalactamases. Cefiderocol shows high in vitro potency against pathogenic carbapenem-resistant Gram-negative bacteria, with the MIC lower than $4 \mathrm{mg} / \mathrm{L}$ for most isolates in Enterobacteriaceae, $P$. aeruginosa and $A$. Baumannii. It has been approved by the Food and Drug Administration (FDA) for the treatment of nosocomial pneumonia and complicated urinary tract infections. Although cefiderocol is a promising antimicrobial agent against MDR Gram-negative bacteria, its efficacy in treating infections caused by carbapenem-resistant $P$. aeruginosa and $A$. Baumannii remains uncertain. And the emergence of resistance has already been reported. Therefore, to promote a more rational use of cefiderocol and to reduce the occurrence of resistance, a deep understanding of this novel siderophore-cephalosporin is quite necessary.

Condition being studied: Cefiderocol for the Treatment of Multidrug-Resistant Gram-Negative bacteria. Three authors participate in the study, two reviewers independently performed the literature search and the study selection. Any disagreement was resolved by a third reviewer, and a final consensus was reached among all authors.

\section{METHODS}

Search strategy: The search terms included 'cefiderocol', 'S-6492660' and 'Fetroja'. Besides, to reduce publication bias, we reviewed the conference proceedings of the International Symposium on Antimicrobial Agents and Resistance (ISARR), Infectious Diseases Society of America (IDSA) and European Congress of Clinical Microbiology and Infectious Diseases (ECCMID) from the years 20152021. We manually searched the reference lists of included studies and published reviews and systematic reviews for selecting relevant articles.

Participant or population: This systematic review including in vitro studies, in vivo animal studies and clinical trails, et al.

Intervention: Studies on cefiderocol. For the clinical studies, the intervention is cefiderocol treatment.

Comparator: For the clinical studies, the comparators are other treatment strategies.

Study designs to be included: Studies were considered eligible for inclusion if they reported cefiderocol on the in vitro or in vivo antimicrobial activity, pharmacokinetics and pharmacodynamics, 
clinical usage (case reports, case series and clinical trails) and resistance mechanisms.

Eligibility criteria: Studies were considered eligible for inclusion if they reported cefiderocol on the in vitro or in vivo antimicrobial activity, pharmacokinetics and pharmacodynamics, clinical usage and resistance.

Information sources: We systematically searched the PUBMED, EMBASE and Cochrane Library databases. Besides, to reduce publication bias, we reviewed the conference proceedings of the International Symposium on Antimicrobial Agents and Resistance (ISARR), Infectious Diseases Society of America (IDSA) and European Congress of Clinical Microbiology and Infectious Diseases (ECCMID) from the years 2015-2021. We manually searched the reference lists of included studies and published reviews and systematic reviews for selecting relevant articles.

Main outcome(s): For in vitro studies on the antimicrobial activity, the primary end-point was the MICs, the susceptible rates. For clinical studies and trials, the primary endpoint was the clinical response and allcause mortality.

Additional outcome(s): The PK/PD targets for cefiderocol, the adverse effects, the resistance mechanisms.

Data management: The following data were extracted by two independent reviewers using standard forms: authors, publication year, details of the experimental methods or study design, number of tested strains, animals or patients, main characteristics of tested strains or the study population and information on outcome measures.

Quality assessment / Risk of bias analysis: For the clinical trials, the Cochrane risk of bias tool was applied to assess the risk of bias of each included study.

Strategy of data synthesis: For data satisfying quantitative analysis, the analyses were performed with Stata 14.0 (Stata Corporation, College Station, TX). For the meta-analysis of clinical trials, risk ratio (RR) and the associated $95 \%$ confidence intervals $(\mathrm{Cl})$ were used as the effect measures for the outcomes. Statistical heterogeneity among studies was assessed by using 12 index ( $12>50 \%$ was considered to be a substantial degree of heterogeneity among studies). The random-effect model was used when the heterogeneity was significant; in all other cases, the fixed effect model was used.

Subgroup analysis: For the in vitro studies, the subgroup analysis will be performed by different classes of bacteria. For the clinical studies, the subgroup analysis will be performed by different infection sites and different infectious pathogens.

Sensitivity analysis: The sensitivity analysis will be performed by removing low quality studies.

\section{Language: English.}

Country(ies) involved: China.

Keywords: cefiderocol, MultidrugResistant, Gram-Negative bacteria, In vitro activity, PK/PD, treatment, resistance mechanisms

Dissemination plans: Publish the systematic review in relevant journals.

\section{Contributions of each author:}

Author 1 - Chuanhai Wang - Author 1 searches the literature, extracts the data, analyzes the data, and drafts the manuscript.

Email: wangchuanhai3420@126.com

Author 2 - Deqing Yang - Author 2 searches the literature, extracts the data, analyzes the data, and drafts the manuscript. Email: ydq18287276341@126.com

Author 3 - Wentao Ni - Author 3 analyzes the data, and drafts the manuscript. Email: wentao.qingdao@163.com 